\title{
Colite necrohemorrágica causada por Entamoeba histolytica em um cão
}

\author{
Necro-hemorrhagic colitis caused by Entamoeba histolytica in a dog \\ Maria Talita Soares Frade', Eduardo Melo Nascimento', Robério Gomes Olinda', Raquel Annes Fagundes \\ Silva ${ }^{2}$, Fabrício Marcus Silva Oliveira ${ }^{3}$, Marcelo Vidigal Caliari ${ }^{3}$ \& Antônio Flávio Medeiros Dantas ${ }^{4}$
}

\begin{abstract}
Background: Intestinal amebiasis with morphological lesions and clinical manifestations is uncommon in dogs. The disease is caused by the protozoan Entamoeba histolytica, which is commonly observed in its natural hosts, humans and some nonhuman primates. It is occasionally found in the company of animals, usually associated with contact with infected humans. Thus, the objective here is to describe a case of necro-hemorrhagic colitis caused by E. histolytica in a dog infected with the canine distemper virus, in order to characterize the epidemiological and clinicopathological aspects of the disease.

Case: An adult, mixed-breed bitch displaying anorexia and ataxia was referred to the veterinary hospital for treatment. Clinical evaluation showed a cachectic animal with $12 \%$ dehydration, ocular discharge, and bilateral purulent nasal discharge. A clinical diagnosis of distemper was made, and treatment was instituted. The dog's signs progressed to walking in circles, aimlessly, with lethargy and blindness. After three days of the onset of neurological signs, the dog developed diarrhea with hematochezia. With no improvement noted, we elected to euthanize the dog. At necropsy, edema was present in subcutaneous tissues, and the lungs had yellow areas in the cranio-ventral portions, which the court was flowing purulent discharge. In the large intestine, segmental distention of the distal portion of the descending colon was observed. The segment was approximately $15 \mathrm{~cm}$ in length and consisted of irregular reddish areas. There was also slight thickening of the wall with edematous mucosa containing blood clots, fibrin, and multiple areas of ulceration. Microscopically showed necro-hemorrhagic colitis associated with rounded structures, approximately $15 \mu \mathrm{m}$ in size, containing abundant eosinophilic cytoplasm that was slightly granular or vacuolated. They also contained nuclei and nucleoli that were central or slightly eccentric. These organisms were consistent with amoeba trophozoites. There was also demyelinating encephalitis associated with malacia, corpuscular intranuclear eosinophilic inclusions and / or intracytoplasmic inclusions in ependymal cells, astrocytes, and gemistocytes, characteristic of infection with canine distemper virus. Using immunohistochemistry with polyclonal anti-E. histolytica antibodies in the dilution of 1:1000, trophozoites were immunomarked, confirming the suspected amebiasis.

Discussion: The diagnosis of intestinal amebiasis was based on clinical signs and by morphological characteristics on gross and microscopic examination, and was confirmed as E. histolytica by immunohistochemistry. Limited information on the epidemiology and pathological findings of infection with Entamoeba sp. has been reported in the literature, as it is relatively uncommon in pets. Affected animals are usually asymptomatic, but immunosuppression caused by canine distemper virus may have triggered the clinical manifestations of the disease in this dog. Enteritis due Entamoeba sp. should be considered in dogs with chronic weight loss and bloody diarrhea. It should also be included in the differential diagnoses for weight loss and hemorrhagic gastroenteritis, such as canine parvovirus, canine adenovirus 1, Pythium insidiosum, and Giardia sp.
\end{abstract}

Keywords: intestinal amebiasis, hematochezia, coinfection.

Descritores: amebíase intestinal, hematoquezia, co-infecção. 


\section{INTRODUÇÃO}

Entamoeba histolytica é um protozoário do filo Sarcomastigophora, classe Sarcodina [20], capaz de invadir a mucosa intestinal e causar disenteria amebiana principalmente em humanos e em primatas não humanos [8].

A doença ocorre após a invasão da mucosa intestinal pelos trofozoítos, e a severidade é influenciada pela dieta, estado imunológico, estirpe e virulência do agente $[13,16]$. Na medicina veterinária a amebíase intestinal é mais frequentemente relatada em primatas de cativeiro [17] e ocasionalmente em cães e gatos [14], normalmente associada ao contato com humanos infectados, já que os animais de companhia não eliminam a forma infectante pelas fezes [17].

A infecção ocorre após a ingestão de cistos através de alimentos ou água contaminados por fezes de humanos portadores [16]. Os trofozoítos móveis são liberados e invadem a mucosa colônica, causando severas lesões ulcerativas e necrosantes na mucosa [8].

Esse relato descreve os achados epidemiológicos e clínicopatológicos de um caso de colite necrohemorrágica causada por Entamoeba histolytica em canino infectado com o vírus da cinomose.

\section{CASO}

Uma cadela errante, sem raça definida, adulta, com histórico de anorexia e ataxia foi atendida na Clínica Médica de Pequenos Animais, do Hospital Veterinário, da Universidade Federal de Campina Grande, Campus de Patos, PB. No exame clínico foi observado escore corporal ruim, desidratação de $12 \%$ e secreção purulenta ocular e nasal bilateral. Em seguida, foi estabelecido diagnóstico clínico de infecção pelo vírus da cinomose e instituído o tratamento sintomático. Após seis dias, o animal manifestou outros sinais neurológicos caracterizados por sonolência, cegueira, andar em círculos e a esmo. No nono dia foi observada diarreia semi-pastosa sanguinolenta. O quadro clínico evoluiu para decúbito lateral e optou-se pela eutanásia.

Na necropsia observou-se estado corporal ruim e com edema no subcutâneo. Nos pulmões havia áreas amareladas irregulares e consolidadas nos lobos cranioventrais, que ao corte fluía exsudato purulento. $\mathrm{Na}$ porção final do cólon descendente foi observada marcada distensão segmentar de aproximadamente $15 \mathrm{~cm}$ de comprimento e a serosa avermelhada e levemente granular. A parede apresentava-se discretamente espessada e a mucosa edemaciada com coágulos de sangue, fibrina e múltiplas áreas com ulcerações (Figura 1A). Fragmentos de órgãos das cavidades abdominal e torácica, e sistema nervoso central foram fixados em formol tamponado a $10 \%$, clivados e processados rotineiramente para avaliação histopatológica pela coloração de hematoxilina e eosina (HE). Microscopicamente verificou-se colite necrohemorrágica, focalmente extensa e acentuada (Figura 1B). Observaram-se também hiperplasia e dilatação das vilosidades intestinal (Figura 1C). Nessas áreas foram observadas estruturas arredondadas de citoplasma abundante eosinofílico, levemente granular ou vacuolizado, com núcleo e nucléolo central, que mediam aproximadamente $15 \mu \mathrm{m}$ de diâmetro, vistas principalmente nas áreas de necrose, características de trofozoítos amebianos (Figura 1D). Na lâmina própria observou-se também discreto infiltrado de eosinófilos, além de discreta necrose e hemorragia na submucosa.

As lesões características de cinomose foram observadas principalmente na substância branca do cerebelo. Observaram-se áreas multifocais de desmielinização associadas à gemistócitos, astrócitos hipertróficos e malacia. Corpúsculos de inclusões eosinofílicos intranucleares e/ou intracitoplasmáticos em astrócitos, gemistócitos e células ependimárias foram evidenciados. Em núcleos da base foram observados discretos manguitos perivasculares multifocais constituídos por infiltrado inflamatório de linfócitos, macrófagos e plasmócitos. Verificou-se também broncopneumonia necrossupurativa, multifocal a coalescente, acentuada. Corpúsculos de inclusões também foram vistos nas células epiteliais da mucosa gástrica e na glândula salivar.

Secções de cólon foram selecionadas para realização de imuno-histoquímica (IHQ) com anticorpo policlonal anti-E. histolytica na diluição de 1:1000, utilizando anticorpo secundário estreptavidina-biotina-peroxidase ${ }^{1}$, revelado com a solução de diaminobenzidina $0,05 \%$ em $\mathrm{H}_{2} \mathrm{O}_{2} 40 \mathrm{vv}$ à $0,2 \%$ e contracorado com hematoxilina de Harris, ocorrendo imunomarcação positiva dos trofozoítos em coloração amarronzada (Figura 1E).

\section{DISCUSSÃO}

O diagnóstico de amebíase intestinal foi baseado nos achados clínicos, macroscópicos e microscópicos, sendo estabelecido amebíase por Entamoeba sp. pelas características morfológicas do protozoário e confirmado E. histolytica pela imuno-histoquímica.

Infecções por E. histolytica em cães e gatos, são geralmente assintomáticas [5]. O estado de portador inaparente e consequentemente com manifestações 


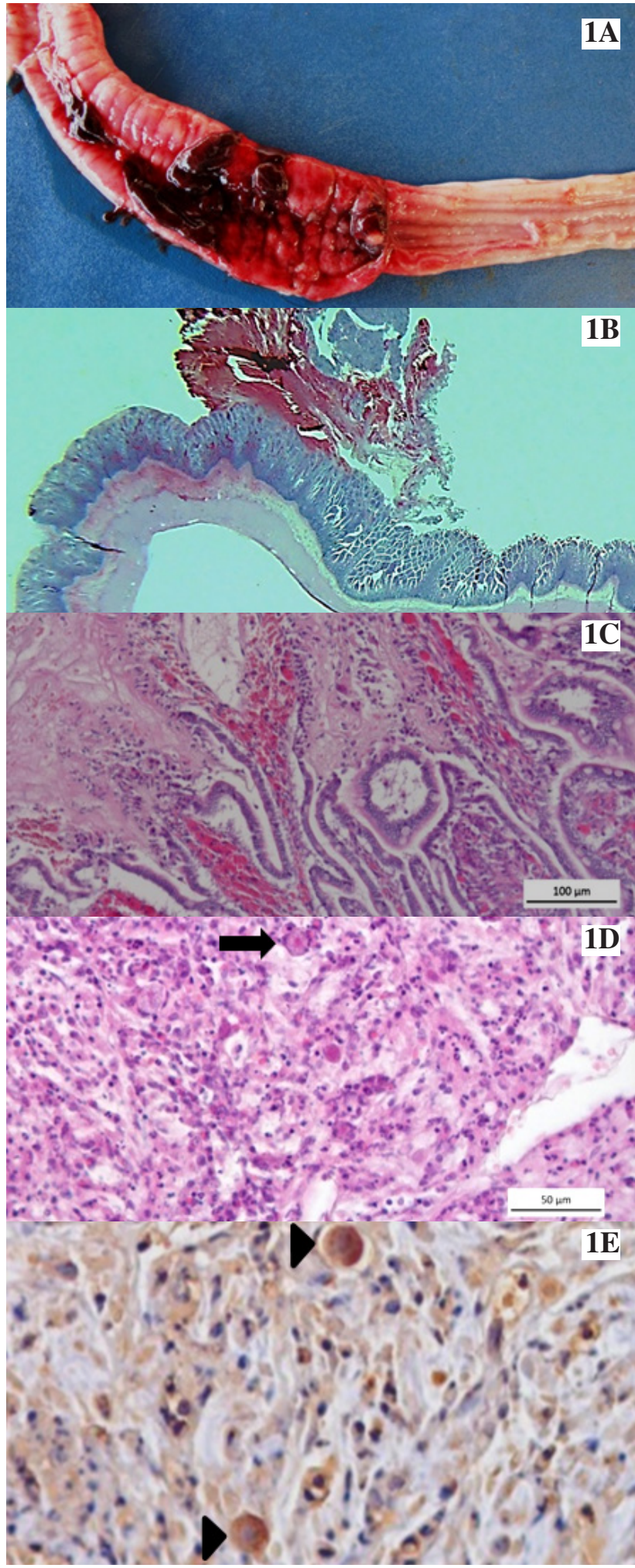

Figura 1. Colite por Entamoeba histolytica em cão. A- Cólon descendente. Observa-se mucosa edemaciada com áreas avermelhadas irregulares associadas a coágulos. B- Submacroscopicamente. Observa-se espessamento segmentar da mucosa e coágulo sanguíneo. C- Observa-se necrose, hemorragia, hiperplasia e dilatação das vilosidades intestinais. [HE, Barra $=100 \mu \mathrm{m}]$. D- Observa-se trofozoíto em área de necrose associado à infiltrado inflamatório na lâmina própria da mucosa intestinal (seta). [HE, Barra $=50 \mu \mathrm{m}$ ]. E- Mostrando múltiplos agregados de grânulos amarronzados distribuídos no citoplasma dos trofozoítos (cabeças de seta), caracterizando imunomarcação positiva para Entamoeba histolytica. Técnica de imuno-histoquímica, método da estreptavidinabiotina-peroxidase contracorados com Hematoxilina de Harris [IHQ, 40x]. clínicas severas, dependem da virulência da estirpe de E. histolytica, espécie hospedeira, estado nutricional, imunológico e microflora intestinal, bem como a presença de patógenos intestinais concomitantes $[15,18]$. No presente caso, o provável fator desencadeante foi à infecção concomitante com o vírus da cinomose canina. Há inúmeros relatos que descrevem a infecção pelo vírus da cinomose como predisponente a infeções oportunistas secundárias em cães, devido a depleção linfoide [2$4,7]$. No entanto, não foram encontrados relatos de co-infecção do vírus da cinomose e E. histolytica em cães.

Animais portadores não infectam outros animais nem o homem, pois excretam a forma trofozoítica que é frágil e não infectante. O homem, no entanto, excreta os cistos que são altamente resistentes e infectantes para as espécies de animais de companhia [5]. Provavelmente esse animal adquiriu a infecção após ingestão de água e/ou alimentos contaminados com cistos. Os animais errantes podem estar mais predispostos à ocorrência da doença, pela maior exposição a regiões com baixas condições higiênico-sanitárias.

Quando não há um tratamento adequado, a colite amebiana é extremamente severa, podendo rapidamente desenvolver ulcerações e por vezes atingir todo o cólon $[1,10]$. Neste caso, as lesões estavam restritas ao cólon descendente, porém, além de comprometer o cólon, trofozoítos de E. histolytica podem ocasionalmente penetrar a submucosa intestinal, atingindo a circulação sanguínea e disseminar-se para outros órgãos, como o fígado [6].

Enterite por Entamoeba sp. deve ser considerada em cães com quadro clínico de perda de peso e diarreia sanguinolenta de evolução crônica. Os sinais clínicos nesse caso foram inespecíficos. Quadro semelhante de diarreia com sangue pode ser observado na forma entérica da parvovirose canina [11], em casos de hepatite infecciosa canina [9] e também na forma gastrointestinal da pitiose em cães [19], devendo ser considerado a epidemiologia e outros sinais clínicos associados para diferenciá-las.

Neste caso, as características histopatológicas e a imunomarcação positiva foram determinantes para o diagnóstico de colite amebiana, constituindo importantes ferramentas de diagnóstico laboratorial.

\section{MANUFACTURER}

${ }^{1}$ Zymed Laboratories Inc. San Francisco, CA, USA.

Acknowledgements. Laboratórios de Amebíase e Protozooses da Universidade Federal de Minas Gerais.

Declaration of interest. The authors report no conflict of interest. The authors are responsible for the content and writing of the paper. 


\section{REFERENCES}

1 Abd-Allá M.D. \& Ravdin J.L. 2002. Diagnosis of amoebic colitis by antigen capture ELISA in patiens presenting with acute diarrhoea in Cairo, Egypt. Tropical medicine \& international health. (7): 365-370.

2 Fawi M.T., Tag El Din M.T. \& El-Sanousi S.M. 1971. Canine distemper as a predisposing factor for Nocardia asteroides infection in the dog. Veterinary Record. 88(13): 326-328.

3 Frade M.T.S., Melo L.F., Pessoa C.R.M., Araújo J.L., Fighera R.A., Souza A.P., Uzal F. \& Dantas A.F.M. 2015. Systemic acanthamoebiasis associated with canine distemper in dogs in the semiarid region of Paraíba, Brazil. Pesquisa Veterinária Brasileira. 35(2): 160-164.

4 Frade M.T.S., Maia L.A., Andrade R.L.F.S., Alves R.C., Yamasaki E.M., Mota R.A. \& Dantas A.F.M. 2015. Clinical, pathological, and immunohistochemistry characterization of toxoplasmosis in dogs with distemper in the semiarid region of Paraíba, Brazil. Semina: Ciências Agrárias. 36(6): 4251-4256.

5 Greene C.E. 2012. Infectious diseases of the dog and cat. 4.ed. Rio de Janeiro: Elsevier, 1376p.

6 Haque R., Huston C.D., Hughes M., Houpt E. \& Petri Jr. W.A. 2003. Amebiasis. New England Journal of Medicine. (348): 156-1573.

7 Headley S.A. \& Saito T.B. 2003. Simultaneous canine distemper encephalitis and canine parvovirus infection with distemper-associated cardiac necrosis in a pup. Ciência Rural. 33: 1149-1151.

8 Huston C.D. 2004. Parasite and host contributions to the pathogenesis of amebic colitis. Trends in Parasitology. 20: 6-23.

9 Inkelmann M.A., Rozza D.B., Fighera R.A., Kommers G.D., Graça D.L., Irigoyen L.F. \& Barros C.S.L. 2007. Hepatite infecciosa canina: 62 casos. Pesquisa Veterinária Brasileira. 27(8): 325-332.

10 Natarajan A., Souza R.E., Lahoti N.G. \& Candrakala S.R. 2000. Ruptured liver abscess with fulminant amoebic colitis: a case report with review. Tropical gastroenterology: official journal of the Digestive Diseases Foundation. 21(4): 201-203.

11 Rodrigues A.F. 2011. Estudo retrospectivo da parvovirose canina diagnosticada no LPA do HV da UFCG do município de Patos-PB (2003-2010). 39f. Patos, PB. Monografia (Trabalho de Conclusão de Curso em Medicina Veterinária) Universidade Federal de Campina Grande.

12 Santos F.L.N. \& Soares N.M. 2008. Mecanismos fisiopatogênicos e diagnóstico laboratorial da infecção causada pela Entamoeba histolytica. Jornal Brasileiro de Patologia e Medicina Laboratorial. 44(4): 249-261.

13Sharma M., Vohra H. \& Bhasin D. 2005. Enhanced pro-inflammatory chemokine/ cytokine response triggered by pathogenic Entamoeba histolytica: basis of invasive disease. Parasitology. 137(6): 783-796.

14 Shimada A., Muraki Y., Awakura T., Umemura T., Sanekata T., Kuroki T. \& Ishihara M. 1992. Necrotic colitis associated with Entamoeba histolytica infection in a cat. Journal Comparative Pathology. 106: 195-199.

15 Stanley Jr. S.L. \& Reed S.L. 2001. Microbes and microbial toxins: Paradigms for microbial-mucosal infections. VI. Entamoeba histolytica: parasite-host interactions. American Journal of Physiology. Gastrointestinal and liver physiology. 280(6): 1049-1054.

16Stanley Jr. S.L. 2003. Amoebiasis. The lancet. (361): 1025-1034.

17 Stedman N.L., Munday J.S., Esbeck R. \& Visvesvara G.S. 2003. Gastric Amebiasis Due to Entamoeba histolytica in a Dama Wallaby (Macropus eugenii). Veterinary Pathology. 40(3): 340-342.

18Toft J.D. 1982. The Pathoparasitology of the Alimentary Tract and Pancreas of Nonhuman Primates: A Review. Veterinary Pathology. 19(7): 44-92.

19 Trost M.E., Gabriel A.L., Masuda E.K., Fighera R.A., Irigoyen L.F. \& Kommers G.D. 2009. Aspectos clínicos, morfológicos e imuno-histoquímicos da pitiose gastrintestinal canina. Pesquisa Veterinária Brasileira. 29(8): 673-679.

20 Urquuhart GM., Armour J., Duncan J.L., Dunn A.M. \& Jennings FW. 2008. Parasitologia Veterinária. 2.ed. Rio de Janeiro: Guanabara Koogan, 285p. 